\title{
JAUMANN, Herbert, Die europäische Gelehrtenrepublik im Zeitalter des Konfessionalismus. The European Republic of Letters in the Age of Confessionalism
}

Anne Saada

\section{OpenEdition}

Édition électronique

URL : http://journals.openedition.org/ifha/1035

DOI : 10.4000/ifha.1035

ISSN : 2198-8943

Éditeur

IFRA - Institut franco-allemand (sciences historiques et sociales)

Référence électronique

Anne Saada, « JAUMANN, Herbert, Die europäische Gelehrtenrepublik im Zeitalter des Konfessionalismus. The European Republic of Letters in the Age of Confessionalism », Revue de I'IFHA [En ligne], Date de recension, mis en ligne le 01 janvier 2004, consulté le 22 septembre 2020. URL : http:// journals.openedition.org/ifha/1035; DOI : https://doi.org/10.4000/ifha.1035

Ce document a été généré automatiquement le 22 septembre 2020.

(CIFHA 


\title{
JAUMANN, Herbert, Die europäische Gelehrtenrepublik im Zeitalter des Konfessionalismus. The European Republic of Letters in the Age of Confessionalism
}

\author{
Anne Saada
}

Ce recueil, issu d'un colloque qui s'est tenu à Wolfenbüttel en juin 1997, traite du rapport entre la République des Lettres, envisagée sous un angle théorique, et sa réalisation dans une Europe déchirée par les conflits confessionnels. Les contraintes et pratiques qui découlaient de l'appartenance confessionnelle étaient-elles compatibles avec la recherche d'une pensée libérée de tout préjugé et avec l'idéal de tolérance prônés par la République des Lettres ? À quels types de pratiques ces exigences a priori contradictoires ont-elles conduit? Telles sont les interrogations qui parcourent les onze contributions qui composent ce volume, rédigées pour certaines en anglais, pour d'autres en allemand et pour l'une en français. Celle de l'éditeur tout d'abord - $\mathrm{H}$. JAUMANN - propose une synthèse critique des recherches sur la République des Lettres : nombre de travaux ne traitent pas directement de la République des Lettres, alors que leurs titres auraient tendance à le suggérer. Aussi, pour éviter cet écueil, l'auteur se lance-t-il à la recherche de la définition exacte de l'objet de cette République des Lettres. A. GRAFTON s'intéresse à la description de la maison de Salomon dans La nouvelle Atlantide, utopie non achevée de Bacon - l'un des fondateurs de la République des Lettres - et montre ce qu'elle doit aux idées et aux pratiques de l'érudition humaniste, et en particulier au projet d'une nouvelle histoire de l'Église développée et réalisée par le théologien luthérien Flacius Illyricus. A. SHELFORD traite des divisions confessionnelles à l'intérieur de la République des Lettres en exposant les conflits de nature intellectuelle qui mirent aux prises l'Académicien catholique Pierre-Daniel Huet avec les huguenots de son temps. L'article de P. MILLER a pour objet la confection de la Bible polyglotte de Paris, qui repose sur la coopération entre des traditions 
scientifiques et érudites hétérogènes. À partir de l'exemple du savant et prêtre italien Muratori, F. WAQUET montre les difficultés - parfois difficilement surmontables rencontrées par un érudit partagé entre les contraintes issues de la foi et les exigences de la science. La contribution de A. GOLDGAR a pour thème l'exil, qu'elle traite à partir de l'exemple du réfugié Jean Barbeyrac - un juriste d'abord, puis traducteur et commentateur. Elle montre comment ce phénomène a contribué au développement de la République des Lettres, dans la mesure où l'exil renforce le sentiment de la communauté d'une part et, d'autre part, contraint à déployer d'importants réseaux de communication pour rester en contact avec les siens. M. VÖLKEL et C. FASOLT présentent chacun un aspect de l'humanisme tardif en Allemagne, en traitant les rapports entre confession et érudition respectivement chez Marx Welser et Hermann Conring. M. SCATTOLA, pour sa part, expose et analyse la controverse entre le philosophe Johannes Caselius et le théologien de Helmstedt Daniel Hofmann. Il montre que les vérités issues des deux disciplines sont difficilement compatibles l'une avec l'autre. La contribution de R. HÄFNER porte sur l'exégète et orientaliste de Helmstedt Hermann von der Hardt, une figure controversée de la philologie. L'article qui clôt cet ouvrage, de G. VOGT-SPIRA, examine dans une perspective confessionnelle la réception de la poétique de l'auteur catholique Marco Girolamo Vida dans l'Allemagne protestante. L'auteur analyse les raisons pour lesquelles le facteur confessionnel est, contrairement à ce que l'on aurait pu attendre, peu intervenu sur la réception importante - de cet auteur.

2 Cet ouvrage, stimulant dans l'ensemble, vient bien compléter les travaux entrepris par H.J. sur la République des Lettres et la culture savante à l'ère de la confessionnalisation. Il incite, comme l'auteur le dit lui-même, à poursuivre les recherches dans une perspective d'historicisation toujours plus poussée de la République des Lettres.

Anne SAADA (Centre National de la Recherche Scientifique) 